\title{
Experiences of patients living with inflammatory bowel disease in rural communities
}

\author{
Rebecca K. Britt, ${ }^{1}$ Andrew Englebert ${ }^{2}$ \\ ${ }^{1}$ Department of Journalism and Creative Media, University of Alabama, Tuscaloosa, AL; ${ }^{2}$ AME Family Law, Menasha, WI, USA
}

\begin{abstract}
Inflammatory bowel disease (IBD) is a chronic disease that often has fluctuating and painful symptoms. IBD patients must cope with a lifelong illness with relapses, remissions, and varied treatments that can affect their overall quality of life. Patients living in a rural setting are faced with further challenges such as access to healthcare, physician availability, and socioeconomic factors. For the current study, we interviewed adult patients in a clinic who were diagnosed with IBD for at least 3 years to better understand their experiences with the aim to inform intervention and educations for patients and physicians. Through a thematic analysis, we argue that five themes emerged from the data: i) IBD etiology, ii) ceding self-care, iii) environmental factors associated with disclosure, iv) stigma, and $\mathrm{v}$ ) environmental obstacles to care. We suggest opportunities for research and collaboration among researchers and practitioners to help reduce stigma associated with IBD and promote health among rural communities.
\end{abstract}

\section{Introduction}

Inflammatory bowel disease (IBD) is a chronic and incurable condition involving inflammation of the digestive tract. IBD often involves severe pain, fluctuating and

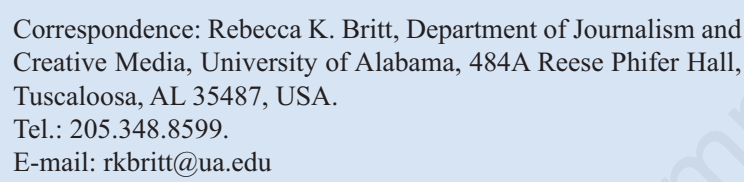

Acknowledgments: the authors would like to thank patients for taking the time to participate in this study.

Key words: Inflammatory bowel disease; Thematic analysis; Health experience; Inflammatory bowel disease in adults; Rural settings.

Contributions: RKB: data collection and analysis, manuscript write-up, manuscript editing; AE: research assistance, assistance with data collection and analysis, literature review, manuscript editing.

Conflict of interest: the authors declare no potential conflict of interest.

Funding: none.

Received for publication: 2 December 2018.

Revision received: 19 March 2019.

Accepted for publication: 19 March 2019.

This work is licensed under a Creative Commons Attribution NonCommercial 4.0 License (CC BY-NC 4.0).

(C) Copyright R.K. Britt and A. Englebert, 2019

Licensee PAGEPress, Italy

Qualitative Research in Medicine \& Healthcare 2019; 3:40-46

doi:10.4081/qrmh.2019.7962 painful symptoms that can result in abdominal pain and even surgery, and can lead to life-threatening situations. ${ }^{1}$ Moreover, IBD is widespread: it affects approximately 1.4 million people living in the United States with about 30,000 new cases diagnosed yearly. ${ }^{2,3}$ IBD affects both men and women, with symptoms affecting the digestive tract in often debilitating manners. The costs of treatment range from varying medications to treat symptoms, to surgery when medications can no longer adequately control symptoms. Individuals with IBD may often appear to be physically well because IBD does not manifest externally, so the difficulties associated with managing the diseases can be further complicated. IBD affects patients in most aspects of their daily lives, such as family, work, friendships and extracurriculars. ${ }^{4-6}$ By understanding the experience of patients living with IBD in areas where health disparities persist, ${ }^{4}$ such as rural communities, we can gain valuable insight on factors that contribute to their quality-of-life.

Among the studies that have explored these issues, patients have communicated anxiety surrounding health care provider interactions, expressing concerns that they tend to treat symptoms rather than the whole problem. ${ }^{7}$ Other studies have found that meaningful dialogue about IBD tends to take place within social support groups ${ }^{8,9}$ which help patients share life experiences, information and knowledge in addition to other social interactions. ${ }^{10,11}$ If we hope to develop appropriately tailored programs to work with providers, we need to understand the unique experiences that rural patients with IBD face. The goal of this research study is to explore the illness experience of patients living with IBD in a rural setting through their accounts given via interviews. Interviews analyzed through methods such as thematic analysis ${ }^{12,13}$ offer an opportunity to better understand how individuals make sense of IBD in a rural setting. ${ }^{12,14,15}$ These experiences can help 
identify and address the struggles of chronic health care in rural communities. By learning about the experiences of individuals who cope with chronic health issues, we as scholars and educators can build strategies that can be used to improve potential barriers in health services that may impede overall health care.

\section{Literature review}

\section{Health care and Inflammatory bowel disease}

IBD primarily consists of chronic inflammation of the digestive tract and is typically diagnosed as Crohn's disease or ulcerative colitis. The diseases commonly have symptoms including abdominal pain and fluctuating bowel symptoms. ${ }^{16}$ IBD illnesses are commonly associated with inflammation of the gastrointestinal tract, and those such as Crohn's disease are typically diagnosed as autoimmune disorders. ${ }^{1,16}$ Similarly, colitis occurs when the colon becomes inflamed and develops ulcers, resulting in abdominal pain. In both cases, symptoms associated with IBD tend to fluctuate. Severe cases of IBD result in hospital care and surgery for the bowels, intestines, colectomies, or proctocolectomy, in which both the colon and rectum are removed. ${ }^{17}$ While diagnosis of IBD can occur at any time, people tend to be more frequently diagnosed between the ages of 15 and 35. ${ }^{2,18}$ IBD affects both men and women; colitis is slightly more common among men, and Crohn's disease is more frequent in women. ${ }^{19}$ As symptoms fluctuate in IBD, a person may experience periods of remission, followed by relapse with subsequent and possibly new forms of treatment, which often vary. ${ }^{19-}$ ${ }^{21}$ As treatments can be complex, health care professionals may struggle to treat patients, ${ }^{22}$ and in other cases, it can be difficult for physicians to diagnose whether or not a treatment is effective for a patient. ${ }^{16,23-25}$

\section{Illness experiences and chronic health diseases}

Listening to a person's experience of living with IBD can provide education and evidence for health care provision and making sense of the disease. The illness experience has been characterized as "an experience of disruption in which the life-narrative is suspended." 12 When chronic disease occurs, it can often change the patient's quality of life, resulting in a need for that person to adjust their lifestyle,${ }^{26}$ including family, ${ }^{27-28}$ work, and social life. ${ }^{27}$ Studies on the illness experience of patients who experience chronic diseases have sought to discover how critical situations can disrupt everyday life. ${ }^{27}$ Rich et $a l .^{27}$ explored the quality-of-life of adolescents diagnosed with chronic health conditions by training them to record visual narratives of their illness experiences. They documented their daily lives and recorded personal monologues about their diseases. As they noted, the documentation revealed that the approach enhanced patients' quality-of-life by providing self-examination. More recently, in a qualitative meta-study ${ }^{20}$ of online peer-to- peer support of individuals with chronic illness, findings show that users tend to mobilize to increase knowledge and exchange knowledge about everyday life while also raising awareness about concerns beyond what providers have given.

Qualitative research in particular has lent itself to exploring the illness experience ${ }^{25,28-30}$ by providing inquiry on questions such as: How do people cope with illnesses that have intermittent symptoms? How certain is the medical knowledge with which they receive? How does one cope with day-to-day life as a result of the condition? The uncertainty behind chronic illness, and subsequently, the illness experience, can affect the patient's entire life. ${ }^{30}$ Health-based scholarship can provide valuable inquiry into the illness experience ${ }^{20,25}$ of patients that live with IBD. This can help us better understand the struggles patients face, and likewise, help both scholars and health practitioners develop programs to provide communication strategies to improve quality-of-life outcomes associated with living with chronic diseases like IBD. Given this, the research question for the study is as follows:

RQ1: What is the experience of an adult patient with $I B D$ living in a rural setting and the potential challenges therein?

\section{Materials and Methods}

\section{Research design}

The goal of our study was to interview patients who were currently receiving treatment for IBD to better understand their experiences living with the disease. We selected a qualitative research approach because it offers an inquisitive process ${ }^{25}$ that allows the patients to more fully provide their own insight on making sense of the illness. ${ }^{3}$

\section{Institutional Review Board approval}

The study received Institutional Review Board approval from the university and waivers signed with the local participating clinic, physicians and participating staff. Participants provided written consent to participate in the research. All participants signed three copies of consent forms: one for our team to retain, one for the clinic, and one for the participant to keep.

\section{Recruitment}

We sought individuals who have had IBD at least 3 years, which is criteria used in prior IBD research. ${ }^{7}$ Participants were recruited through a local clinic in a town in a Midwestern part of the United States, with the assistance of clinical staff. No personal medical information was gathered. For our research team to receive confirmation that a patient had been diagnosed with IBD, that patient had to provide consent in a visit with a physician and confirm that they would be willing to participate in a private interview 
about their experiences with IBD. Participants received a $\$ 15$ gift card as incentive for participation.

Our participants were aged between 18 and 51 years old. All participants met the criterion of being diagnosed with IBD more than 3 years prior to the interviews. Of the participants, 21 were women and 6 were men. No participant names were included in interviews or transcription of the audio recordings. Given that participants self-selected to participate, possible implications for the discrepancy of gender are noted in the discussion section.

\section{Data collection}

Our interviews were conducted at the university campus in my office (lead author). This was a careful and deliberate choice, and selected as I have immediate family members who live with IBD, so our team felt I would be able to empathize with patients in the interview process. After careful deliberation, I agreed that this would be an optimal choice. The interview guide was developed by our team, interviews were semi-structured and our questions asked about participants' unique experience with IBD, familial experiences, strengths and weaknesses associated with care (such as healthcare access in rural communities, quality of life), and an open opportunity for participants to share their own stories. Additional topics in our interviews covered treatment experiences, potential stigma associated with IBD, experiences with health care professionals, initial diagnosis, disease knowledge, and experiences associated with support (e.g., family and friends). I strove to ensure that participants' voices were dominant in the course of interviews, so that they could tell their stories. This meant that the interview guide served as a way to direct the conversation by opening spaces for answers to unfold, but that as a researcher, I could remain humble and listen to the stories of patients during this process as opposed to directing these sessions. Generally speaking, the interviews ranged from 40 minutes to 1 hour in length.

\section{Data analysis}

Each interview was transcribed by our team (myself and a research assistant). We employed a thematic analysis approach, drawing upon Braun and Clarke ${ }^{13}$ to code participants' spoken words. Thematic analysis ensures that participants' dialogue about their experiences were directly reflected within our analysis. Thematic analysis involves a careful reading of transcripts, revealing the patterns of shared meanings and experiences across the data. This process first began with us familiarizing ourselves with the data, where we read over the data to get a general understanding of the content. Next, we took detailed notes about the ideas within the data, which helped to create an initial coding structure. We then collapsed the previous ideas into themes. After, we reviewed how the data fit into the themes identified, refining and naming themes to clar- ify our own understanding of the data. ${ }^{13}$ We then reviewed the data a final time with the final themes that emerged.

\section{Results}

Following the results of our thematic analysis, five key themes emerged: i) IBD etiology, ii) ceding self-care, iii) environmental factors associated with disclosure, iv) stigma, and v) environmental obstacles to care. Although findings within themes do overlap, each presented unique content that patients negotiated through coping with experiences associated with IBD.

\section{Inflammatory bowel disease etiology}

To treat IBD, we must know what causes it; but it is complex, chronic, and has multifaceted symptoms that can be difficult for practitioners to make sense of. Few participants had beliefs associated with IBD etiology or knowledge of the disease itself. When I asked about how participants made sense of IBD or defined it, most reported that IBD was a largely hidden disease. In one case, I spoke with an individual who identified as a young, working mother. She noted that IBD was as "weird as it is to discuss it. I've never seen someone that I could pick out with it, just as I never knew what it was before I had it. If you asked me to describe what causes IBD, I couldn't really tell you." Notably, this same young woman explained that she had a difficult time raising her child and managing IBD, in part because she "felt there is a shortage of care... just because I am connected to a doctor and have medicine does not mean there's a solution." While unrelated to the theme of etiology, her story connects to larger concerns associated with IBD knowledge of the disease and service shortages to healthcare in a rural setting (e.g., inpatient substance use and treatment). No participants reported what they believed to be factors that contributed to the onset of IBD, or prior knowledge of the disease. However, participants did report that post-diagnosis, they began to try to make sense of it, though this continued to be a source of uncertainty. The following participant, a man in his mid 40s who was a crop farmer, shared the following excerpt about his understanding of IBD:

I did not know what IBD was. If you told me what Crohn's was, that Colitis is a disease, I would have thought this was a joke! Now I'm hearing pathogen from my doctor and it's not my time to look it up unless it's going to help me.

This same participant mentioned posited that "My doctor, a lot of times, tries to give me as much as possible, even when I don't need the help. At times, she brings up issues I doubt are related. But she's giving me the biggest bang for my buck at each appointment... There are a lot of patients that need treatment." He noted, too, that he "is an optimist...I'm lucky that I own my business. I'm sure there are others who don't get this life." Though IBD knowledge 
may be deprioritized for a participant, it may be that, as this participant at least recounts, physicians may provide integrated services to ensure that patient needs are met. Moreover, participants at times reported uncertainty associated with fluctuating symptoms with IBD, and how they tried to make sense of it, often noting that it was difficult since it was likewise a struggle to make sense of the disease itself (e.g., "If you don't know how to define IBD, well, I think probably it's hard to also communicate about it"). Several participants mentioned that they "... wish I could have prevented this, but I don't know how. I tried to figure it out, but somehow feel as though it was in vain."

\section{Ceding self-care}

In several cases, several participants I spoke with reflected on their present health and coping mechanisms regarding IBD. While some participants described their care of IBD as one that they managed on their own, many participants discussed their decision to cede control of their IBD up to family members and health care professionals. In the following excerpt, a participant, an 18-year-old young woman in college, reported, "I've got enough time to figure out what to eat...whether to get Remicade... it does make me think I can do it, it's in my hands, my choice." She reported that IBD had already interrupted her life during "the point at which I should be living my best life...my best friend, my roommate, she helps me stay on track because I figure it out, sort of." This participant later noted, "You don't know what to do at times, so you just figure it out as you go." As she later noted, "It's just that I already feel stressed out... so I had her help me keep track of my doctor's appointments. I forgot one once, had to pay a fine, and I couldn't get in for a few weeks, and I felt awful. It was a wakeup call for missing something... It's already an hour away. I realized I couldn't do it alone and just needed someone to help me take care of this." Other participants noted that if they forgot an appointment, it was difficult to make another appointment in a similar timeframe. A man in his early 30 s told me that he felt "bad... It's a lot to manage. You have work, life, everything. So, I have my wife help me remember the Flagyl and the next appointment. It's that way with my doctor, I say "Okay" to everything." This participant later discussed that the illness itself remains a struggle. He noted that, "I don't always have a way to describe it- I would not say I'm someone who needs much support, if you do ask, I do not have an accurate description of Crohn's. I'm not sure my doctor does, either."

It is noteworthy, however, that these instances of ceding self-care did not mean that participants merely give up control. Rather, that of ceding self-care to was a decision to negotiate daily care with family members or friends. What we noticed in the data is that this was often spurred by participants' self-awareness, sometimes after missing a doctor's appointment and noting the duration between the next available appointment. Notably, while longer wait times to see a physician not atypical in a given community, the length of time between visits, coupled with struggles associated with transportation reaching health care providers, cost of care, among other challenging factors.

\section{Environmental factors associated with disclosure}

We would like to point out that living in a small community may have benefits and drawbacks for patients. According to most participants I spoke with, having IBD meant that disclosure about their health care, whether with friends, family, or coworkers, often came with struggles. Many participants commonly discussed their experience with IBD in an environmental context, meaning that they contextualized the effects of the disease in reference to activities with local and organizational memberships. One participant I spoke with, a mid 40s woman who was a former healthcare worker for the very clinic she was treated at, noted: "In this town, everyone knows your business." She later told me that for her, "I am on good terms with everyone. But even I have the same wait as everyone else. That's fair. I wish I could tell everyone, "I know you are suffering because I am too." And if I see them in town, and I feel like we are privileged, because anywhere else, you don't know each other." I asked her if she could explain this to me, and she noted, "I'm proud to be from here. We're larger than a very small town. But I run into people and know them by name. I prefer knowing who everyone is." While these comments were not directly related to this participant's unique struggles with IBD, they were related to environmental factors associated with disclosure, and her perceived benefits of living in a smaller, rural community. This participant noted that the ability to more easily know community members by name and learn about them - though she noted this was partially linked to her profession - meant that she felt connected, which would not be as possible in a bigger city.

However, there were other participants I spoke with who felt differently. Some participants tended to describe the difficulties associated with managing symptoms associated with IBD while needing to carefully hide it from their local community, which would be otherwise easier in a larger city. One participant, a 20 -year-old woman, told me, "I'm a runner. I was always the healthy one. It made me [sick] to think that...so no, no, I could never share anything about IBD." She further explained that she meant that she was "always the most fit out of my friends, and honestly, I'm the most competitive...I ran a $5 \mathrm{k}$. I thought I was on my game." She continued by telling me, "IBD made it harder to go on. I trained for $5 \mathrm{ks}$. But not now. Actually, now I can't even find a proper specialist. I have to go to [name undisclosed for review] since there's nobody in the area for the digestive surgery I probably need. It's like a part of you is lost and you can't explain why you don't go train anymore." I listened, and she continued: "But if I could choose, my hometown hospital 
wouldn't be my choice, because I've got this fear that I'd run into someone. And they don't have the knowledge the way that the bigger hospitals do." Several participants shared similar stories, noting that they were concerned about their friends being unempathetic about their disease; in some cases, participants were concerned about some family members lacking concern, given that it can be difficult to make sense of the disease, its symptoms and the pain associated with. An individual with IBD can exhibit symptoms without physically appearing unwell, yet they may still be unable to participate in activities in their local community, so it is unsurprising that many participants express such communication.

\section{Stigma}

In my conversations with participants, most expressed broad concerns with stigma, such as the concern that health care professionals did not take them seriously, particularly if they had fluctuating IBD symptoms. By this, I mean that fluctuating symptoms might include pain, fever, reduced appetite, weight loss, and other symptoms that were expressed. In some cases, concerns associated with stigma included that of family and friends, but by and large were emphasized among healthcare providers. In one conversation with a 45-year-old woman, she discussed her struggles with expressing her concerns about IBD to her doctor: "When you go to the doctor, one day, you might feel alright, and then next time, you're in pain and can't describe it...You might feel like they don't take you seriously after awhile...later, I felt just weird going back, just embarrassed because you don't know what to say since you can't describe each day."

She continued, noting that, "You sometimes even think, you don't want to tell your doctor that you're afraid of having problems with the toilet, even though that's just the idea, but not really going to happen. Maybe if you have an accident, they'll take you seriously." I spoke with other participants who mentioned similar comments, such as my conversation with the young 18 -year-old woman in college, who I referenced earlier (in the theme of ceding self-care), who said, "Even though I want my health taken seriously, it's also embarrassing to even tell my doctor. If your doctor doesn't have it, even they might think it's strange. There's very few people who know beyond my roommate." Other participants made similar comments, that it was difficult to visit their specialists, whether due to symptom fluctuation or multiple treatments, largely because of fears that they would not be taken seriously. I spoke with a 27-year-old man who had spent most of his life working in a labor-intensive agricultural job. He noted that he had to ask his supervisor to adjust his duties after his diagnosis, as the occasional pain from IBD made it too difficult to complete various tasks, but that he had to carefully negotiate how he expressed the symptoms associated with IBD, "because they're just too unusual." He also expressed the toll that IBD took on him, saying, "You can't go to your friends since it's just shamed, and then your doctor thinks you don't take care of yourself." He later continued, saying, "Even now, it's been two years. I am not expressing all those symptoms to a nurse or doctor." The feelings associated with such frustration continued among participants who expressed similar concerns. Perhaps quite poignantly, as the young 18 -year-old woman in college stressed to me, "If IBD was more clearly understood, it wouldn't be something that would be embarrassing to speak about to others."

\section{Environmental obstacles to care}

Perhaps most dominantly associated with challenges associated with rural care is that many participants disclosed the difficulties in accessing necessary services for care, which were often located at greater distances from their local community. These obstacles were likewise hindered by transportation costs to specialty clinics, a lack of private or public transportation available, and wages lost due to illness. Given that IBD care often requires long-term treatment, many participants reported the challenges associated with barriers after diagnosis. For several participants, this meant coping with new treatment when faced with new options that might be expensive or have difficult side effects. I spoke with one participant, a 39-year-old woman who worked as a receptionist for a large manufacturer, who told me that she had to re-negotiate her priorities with work, healthcare, and family life. As she told me, "Once I was diagnosed, I supposed that I had to figure out what my treatment would be. I got sent to a specialist." She continued, saying "I'm normally sitting a lot. But the idea of sitting for an hour in a car and losing that time at work is not realistic. Not for me. I need those hours."

The above story was echoed by other participants I listened to; for instance, participants likewise discussed obstacles to necessary follow-up care for more severe cases associated with IBD, along with struggles due to travel to specialty clinics.

One participant, a former runner (previously mentioned in environmental factors associated with disclosure) discussed her challenges with treatment options. "Cipro and Gengraf weren't available for days. I had to wait and not only that, but I had to pay for it. It's dangerous and feels like that shouldn't happen, but when you're too far from (redacted), it's just the reality." She also told me, "I do think that where you are has a huge impact on the quality of care. I think we have fine doctors, but they can't do much about the special care or the distance." Concerns were also posed about whether or not treatments would continue to be accessible given that new options for care continue to emerge, and that current regimens would be subsequently unavailable. While unsurprising, this is still important to note: access to healthcare facilities, availability of treatments, and challenges with managing work while also receiving care are common issues that patients living in remote or rural areas face. 


\section{Discussion}

Communicating about IBD, whether through feeling comfortable disclosing the diagnosis or communicating the uncertainties it poses and subsequently receiving treatment, results in challenges, which can heavily manifest in rural communities, particularly given the nature of the disease as an invisible illness. ${ }^{20}$ The themes of IBD etiology and ceding self-care were largely associated with participants reporting difficulty in the disruption that IBD had on their lives and the uncertainty it presented. While many participants tended to report a lack of prior knowledge on IBD and uncertainty with managing the disease, they also reflected on receiving help in managing the disease up to others - for example, in the account of a young college student, she relied on the help of a roommate. Our findings have both consistencies and contrasting findings to prior research, which have found that patients, when empowered, strive to take control of their care..$^{5,10}$ As the theme of environmental factors associated with disclosure revealed, participants expressed reluctance to participate in their normal communal activities. IBD is a complex disease by nature, given its fluctuating symptoms, treatment, and nature, so one type of uncertainty that patients face is the complex etiology of the disease. As one patient noted, if the disease was better understood, that might also help to resolve perceived stigma associated with IBD.

When patients feel they cannot adequately communicate with health care providers or loved ones, resistance to treatment or furthering interpersonal relationships can result. We found that participants reported disclosing their disease with some, but not all, family members, along with fears about communicating with health care specialists about IBD. In several instances, participants noted that because of the words associated with IBD - such as bowel or digestive tract, such connotations left them embarrassed. Given the perception of stigma on IBD and its social implications, ${ }^{30}$ this poses challenges for communication, which can certainly impact quality of life. The translational nature of communication about stigmatized illness and reluctance to communicate means there is a need to increase education and empathy associated with the disease. This means that programs that provide education are needed and access to care in rural areas, especially to assist patients like those who participated in the current study. Likewise, we suggest forward-data mining real time communication from parties including the public and health departments (e.g., via Python) to assess how public communication on social media about the disease is actually disclosed. We believe that such studies could yield valuable data that, when paired with qualitative analysis, could result in important insight that could inform the development of a highly tailored educational intervention. Musaev et al. ${ }^{31}$ recently used this approach to explore the communication of state health departments of cardiovascular disease to inform health interventions. We suggest that such an approach should not be used in isolation, but paired with qualitative strategies to engage in the depth of communication and conversations with patients and providers regarding complex, chronic health issues.

Notably, a limitation in the study is that participants were recruited in a single rural community located in the United States. While studying a concentrated region can yield valuable data, these make the findings less generalizable than if interviews were conducted in a broader region, or among multiple teams that can then collate the data and compare findings. Moreover, while unintended, the majority of study participants were female, which may be attributed to self-selection and through their visits with healthcare providers. While the themes that emerged in the study were clearly associated with the concept of experiences associated with IBD and living in a rural community, every effort should be made to include equal gender representation. However, it is notable that the area in which we conducted interviews was rural, with limited access to healthcare resources, which may impact participants' experiences shared.

\section{Conclusions}

It is undeniable that challenges persist associated with the illness experience of IBD. As a result of this study, we encourage scholars to dig deeper into how individuals with chronic health diseases manage illness discomfort and potential variability in health care services, which can affect quality of life. We also encourage scholars to navigate the stigma often associated with IBD and how it influences mental and physical health. While our findings regarding limitations in rural health care to be similar to other studies, ${ }^{4,21}$ a finding of ceding self-care emerged, which suggested that participants' self-awareness impacted their decision making associated with how they cared for their IBD. More attention should be given to patient's decisionmaking strategies associated with IBD and the concept of self-control with complex, chronic illness and the subtle impact of what factors lead to making decisions associated with changes in self-care. The same holds true for stigma, which in this case, largely emerged through our participants largely referencing their communication with a healthcare provider and concerns about stigma on behalf of a physician. While concerns about stigma and IBD was referenced with family and friends, participants reported concerns about being taken seriously by physicians, embarrassment over disclosing the disease, and describing the disease to a physician - even after diagnosis.

This study served to explore the illness experience of patients with IBD living in a rural setting. We found that the challenges associated with treatment of IBD remain a struggle in communities where access to care is often limited, or in some cases, where patients wish for their disease to remain undisclosed. Likewise, we identified that the complexity of IBD resulted in challenges with making 
sense of it, from its etiology to self-care and treatment. We would like to conclude with several final recommendations. First, while it is not necessarily easy, scholars, educators, and practitioners can collaborate to develop education interventions to facilitate knowledge about IBD. Next, we must work to develop patient-centered care to empower those with IBD and improve communication with health care providers. If we develop these partnerships, we can build programs that can promote communication to increase patient self-efficacy and decrease stigma associated with these illnesses. Finally, future research should explore the patient experiences of chronic diseases in the context of self-management, especially since this has grown as an area of national priority, as indicated by the National Institutes of Health. ${ }^{32}$ Ultimately, these future projects might help us serve patients who suffer from chronic diseases.

\section{References}

1. American Gastroenterological Association. What is inflammatory bowel disease? Available from: http://www.gastro. org/info_for_patients/ibd-101-what-is-inflammatory-boweldisease. Accessed: September 2018.

2. Crohn's \& Colitis Foundation. Fact sheet - About IBD. Available from: http://www.crohnscolitisfoundation.org/news/for-themedia/media-kit/fact-sheet-about-ibd.html. Accessed: September 2018.

3. Ng SC, Shi HY, Underwood FE, et al. Worldwide incidence and prevalence of inflammatory bowel disease in the $21 \mathrm{st}$ century: A systematic review of population-based studies. J Lancet 2018;23:2769-78.

4. Smith JD, Margolis SA, Ayton J, et al. Defining remote medical practice: A consensus viewpoint of medical practitioners working and teaching in remote practice. Med J Aust 2008;188:159-61.

5. Björkman I, Simren M, Ringström G, Ung EJ. Patients' experiences of health care encounters in severe irritable bowel syndrome: An analysis based on narrative and feminist theory. J Clin Nurs 2016;25:2967-78.

6. Kennedy A, Robinson A, Rogers A. Incorporating patients' views and experiences of life with IBS in the development of an evidence based self-help guidebook. Patient Edu Couns 2003;50:303-10.

7. Håkanson C, Sahlberg-Blom E, Ternestedt BM. Being in the patient position: Experiences of health care among people with irritable bowel syndrome. Qual Health Res 2010;20: 1116-27.

8. Britt RK. Online social support for participants of Crohn's and ulcerative colitis groups. Health Commun 2017;32:1529-38.

9. McCormick JB, Hammer RR, Farrell RM, et al. Experiences of patients with chronic gastrointestinal conditions: In their own words. Health Qual Life Outcomes 2012;10:1-11.

10. Fletcher PC, Schneider MA, Van Ravenswaay V, Leon Z. I am doing the best that I can! Living with inflammatory bowel disease and/or irritable bowel syndrome (part II). Clin Nurse Spec 2008;22:278-85.

11. Cámara RJ, Lukas PS, Begré S, et al. Effects of social support on the clinical course of Crohn's disease. Inflamm Bowel Dis 2010;17:1277-86.
12. Kleiber DA, Brock SC, Lee Y, et al. The relevance of leisure in an illness experience: Realities of spinal cord injury. J Leis Res 1995;27:283-99.

13. Braun V, Clarke V. Using thematic analysis in psychology. Qual Res Psychol 1006;3:77-101.

14. Goffman E. Stigma. Englewood Cliffs, NJ: Prentice Hall; 1963.

15. Roth J. Timetables. New York: Bobbs-Merrill; 1963.

16. Brittain KR, Shaw C. The social consequences of living with and dealing with incontinence - A careers perspective. Soc Sci Med 2007;65:1274-83.

17. Betteridge JD, Armbruster SP, Maydonovitch C, Veerappan GR. Inflammatory bowel disease prevalence by age, gender, and geographic location in the U.S. military health care population. Inflamm Bowel Dis 2013;19:1421-7.

18. Crohn's \& Colitis Foundation of America. IBD: The basics. Available from:

http://www.crohnscolitisfoundation.org/what-are-crohns-andcolitis/. Accessed: October 2018.

19. Centers for Disease Control and Prevention. Epidemiology of the IBD. Available from: https://www.cdc.gov/ibd/ibdepidemiology.htm. Accessed: October 2018.

20. Kingod N, Cleal B, Wahlberg A, Husted GR. Online peerto-peer communities in the daily lives of people with chronic illness. Qual Health Res 2016;27:89-99.

21. Jones J, Curtin M. Traumatic brain injury, participation, and rural identity. Qual Health Res 2010;20:942-51.

22. Triantafillidis JK, Merikas E, Georgopoulos F. Current and emerging drugs for the treatment of inflammatory bowel disease. Drug Des Devel Ther 2011;5:185-210.

23. Dibley L, Coggrave M, McClurg D, et al. "It's just horrible": a qualitative study of patients' and carers' experiences of bowel dysfunction in multiple sclerosis. J Neurol 2017;264:1354-61.

24. Dixon-Woods M, Critchley S. Medical and lay views of irritable bowel syndrome. Fam Pract 2000;17:108-13.

25. Håkanson C, Sahlberg-Blom E, Nyhlin H, Ternestedt BM. Struggling with an unfamiliar and unreliable body: The experience of irritable bowel syndrome. J Nurs \& Health Care Chron Ill 2009;1:29-38.

26. Dibley L, Norton C, Whitehead E. The experience of stigma in inflammatory bowel disease: An interpretive (hermeneutic) phenomenological study. J Adv Nurs 2018;74:838-51.

27. Rich M, Lamola S, Gordon J, Chalfen R. Video intervention/prevention assessment: A patient-centered methodology for understanding the adolescent illness experience. J Adol Health 2000;27:155-65.

28. Husain A, Triadafilopolous G. Communicating with patients with inflammatory bowel disease. Inflamm Bowel Dis 2004;10:444-50.

29. Altschuler A, Collins B, Lewis JD, et al. Gastroenterologists' attitudes and self-reported practices regarding inflammatory bowel disease. Inflamm Bowel Dis 2008;14:992-99.

30. Taft TH, Keefer L, Leonhard C, Nealon-Woods M. Impact of perceived stigma on inflammatory bowel disease patient outcomes. Inflamm Bowel Dis 2009;15:1224-32.

31. Musaev A, Britt RK, Hayes J, et al. Study of Twitter communications on cardiovascular disease by state health departments. ICWS 2019;1-9.

32. National Institutes of Health. Self-management report. Available from: https://report.nih.gov/NIHfactsheets/ViewFactSheet.aspx?csid $=70$ 